\title{
Electronic Sputtering of Silicon Suboxide Films by Swift Heavy Ions
}

\author{
W. M. Arnoldbik, P. A. Zeijlmans van Emmichoven, and F. H. P. M. Habraken \\ Surfaces, Interfaces and Devices, Debye Institute, Faculty of Physics and Astronomy, Utrecht University, P.O. Box 80.000, \\ NL-3508 TA Utrecht, The Netherlands \\ (Received 4 February 2005; published 24 June 2005)
}

\begin{abstract}
Two regimes are discerned experimentally in the electronic sputtering of silicon suboxide $\left(\mathrm{SiO}_{x}\right)$ films under irradiation with $50 \mathrm{MeV} \mathrm{Cu}$ ions. For $x<1$ the removal rate is low and increases with $x$; for $1.5<$ $x<2$ the removal rate is high and about constant [(1.6-2.0) $\times 10^{3}$ atoms per incoming ion]. The transition occurs at $x$ values where the electronic structure of $\mathrm{SiO}_{x}$ has a transition. We propose a model, which connects the electronic sputter rate with the $\mathrm{SiO}_{x}$ electronic structure considering the lifetime of the ion track.
\end{abstract}

The role of a nonuniform charge distribution in the modification of a solid as resulting from a strong excitation of its electronic system ("Coulomb explosion") is of broad interest. Examples are the modification of a solid by lowenergy highly charged ions (potential sputtering) [1], by intense laser pulses (laser ablation) [2], or by $\mathrm{MeV}$ ions (electronic sputtering) [1,2]. An important issue is the lifetime of the excited state of the solid. In this context we report an experimental study of the electronic sputtering of $\mathrm{SiO}_{x}$ thin films under $\sim \mathrm{MeV} / \mathrm{amu}$ ion irradiation for varying $x$, and discuss how the Coulomb track lifetime varies with the $x$ value and its consequences for the sputtering effect. Indeed, the mechanism of (electronic) sputtering of solids as a result of irradiation by $\mathrm{MeV}$ ions in the energy region of electronic stopping is a matter of continuing debate $[3,4]$. Apart from the interest from the field of ion beam analysis and modification of materials, the study of the process of electronic sputtering has been stimulated by its occurrence in the solar system [5]. The ion-solid interaction starts with energy deposition in a region around the ion path into the electronic system of the solid, giving rise to energetic electrons and a cylindrical track containing excited and positively charged atoms or ions $[4,6,7]$. Obviously, such a track is unstable: chemical bonding is absent, the positively charged ions repel each other, and the high concentration gradient of holes in and/or near the track provokes a strong drift of holes to neutralize the track region. As a result of the existence of the energetic electrons and this "Coulomb track" the material becomes heated ("thermal spike"), and subsequently relaxes in the original or in a different state, giving rise to the socalled latent track. The final state can be different in various respects: chemically, e.g., by formation of inert molecules which may subsequently desorb [8], or structurally, e.g., by an amorphous-crystalline transition or vice versa.

An important question for the understanding of electronic sputtering is whether the nonuniform charge distribution lives long enough to provoke direct desorption of the atoms or ions of the solid ("Coulomb explosion") [9] or that sputtering is an indirect result of modifications of the material. Such modifications could be due to the thermal spike [3], a pressure pulse [10], or an intermediate defect-related mechanism as proposed for potential sputtering by slow highly charged ions $[1,11,12]$. Most studies published so far focused on the dependence of the sputter yield on the energy deposition [13] or compared the electronic sputter yields of widely varying materials $[3,14]$. In contrast, in this Letter, we report the rate of electronic sputtering of amorphous silicon suboxide $\mathrm{SiO}_{x}$ films as a function of $x$ for $0<x \leq 2$. This enables us to investigate, under equal experimental conditions, electronic sputtering of a well-known class of solids that shows a transition from semiconducting to insulating properties. The results are related to the electronic structure of the $\mathrm{SiO}_{x}$ films and appear to be well understood within the framework of a recent molecular dynamics study of Coulomb explosion sputtering $[4,15]$.

The rate of removal of material from $\mathrm{SiO}_{2}$ as a result of irradiation with $\sim 1 \mathrm{MeV} /$ a.m.u. ions is significant $[16,17]$ and large in comparison with that of other oxidic materials [14]. The sputter rate of $\mathrm{SiO}_{2}$ strongly depends on the angle of incidence of the ions and on their stopping power and can be as large as $10^{4}$ atoms per incident ion for $78 \mathrm{MeV}$ $\mathrm{Ag}$ ions at a glancing angle of $7^{\circ}$ [17]. At an equal projectile kinetic energy, the $\mathrm{SiO}_{2}$ removal rate depends on the charge state of the impinging ion, which emphasizes the electronic nature of the sputter rate determining step [17]. The electronic sputter rate of elemental silicon is much lower [18].

The amorphous $\mathrm{SiO}_{x}$ layers were obtained by rf magnetron sputtering of $\mathrm{Si}$ in an $\mathrm{Ar} / \mathrm{O}_{2}$ mixture onto crystalline $\mathrm{Si}$ wafers, dipped in HF before deposition. The $\mathrm{O}$ concentration in the suboxide films was controlled by adjusting the $\mathrm{O}_{2}$ partial pressure [19]. We did not apply additional heating of the substrate. During deposition the temperature slowly rose to a maximum value of $70^{\circ} \mathrm{C}$. The thickness of the films amounted to $80-150 \mathrm{~nm}$.

The samples were irradiated with $50 \mathrm{MeV} \mathrm{Cu}{ }^{8+}$ ions from a 6.5 MV EN tandem accelerator, at a glancing angle 
of incidence of $5^{\circ}$. The particle current density was about $2 \times 10^{11} \mathrm{~cm}^{-2} \mathrm{~s}^{-1}$. The macroscopic temperature of the sample remained below $150{ }^{\circ} \mathrm{C}$. The stopping power of these ions in $\mathrm{SiO}_{x}$ ranges between $7.3(x=0)$ and $8 \mathrm{keV} / \mathrm{nm}(x=2)$, of which only $0.35 \%$ is due to nuclear energy loss. During irradiation the layer thickness and composition were continuously monitored by elastic recoil detection (ERD) using an ionization chamber as the detector [17].

Typical ERD O-concentration depth profiles of a $\mathrm{SiO}_{1.27}$ film during irradiation with $50 \mathrm{MeV} \mathrm{Cu}$ ions are shown in Fig. 1. We note that the bulk $\mathrm{O}$ concentration remains constant, but that the total amount of $\mathrm{O}$ decreases. It should be noted that in the case of $\mathrm{SiO}_{2}$, in situ x-ray photoelectron spectroscopy did not show the formation of suboxide at the surface as a result of irradiation.

Figure 2(a) shows that for every $x$ value the total oxygen content of the layer decreases at a constant rate or remains virtually unchanged with ion fluence. From the slopes and from the experimental observation that $\mathrm{O}$ and $\mathrm{Si}$ are predominantly removed according to the layer composition, we derived the number of atoms $(\mathrm{Si}+\mathrm{O})$ removed per impinging ion, as a function of $x$ [Fig. 2(b)].

The removal rate strongly varies: it decreases from above $1800(\mathrm{O}+\mathrm{Si})$ atoms per incoming ion for $x=2$ down to a value below the sensitivity of $150(\mathrm{O}+\mathrm{Si})$ atoms removed per ion for $x<0.5$. A steep variation is found at about $x=1.2$.

At the same $x=1.2$ a steep variation in the conductivity of the undisturbed material has been shown to occur [20]. In the following we experimentally show that this conductivity has no direct relevance for the magnitude of the rate of electronic sputtering. Since annealing is known to decrease the (variable-range-hopping) conductivity by $2-$

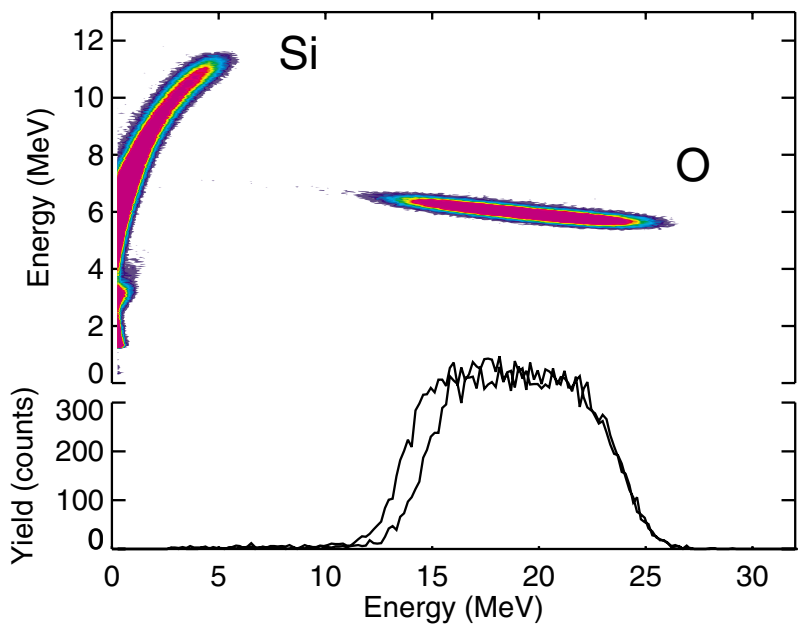

FIG. 1 (color online). Upper part: two-dimensional ERD spectrum. Vertically: energy loss of recoil particle in the first part of the ionization chamber; horizontally, its residual energy. Lower part: projection on final energy axis at beginning and intermediate stages of sputtering for $\mathrm{SiO}_{x}$ film with $x=1.27$.
3 orders of magnitude, we heated these suboxide films at a temperature of $400{ }^{\circ} \mathrm{C}$ for $1 \mathrm{hr}$. This annealing treatment did not result in a significant change of the electronic sputter rate [see Fig. 2(b)], and we conclude that the value of the (defect-related) macroscopic room temperature conductivity does not determine the electronic sputter rate. This is not surprising in view of the massive electronic excitation in a microscopic region around the ion trajectory provoking the existence of many more electronic states contributing to the charge transport than are present as built-in defects in the materials. Consistently, we have measured that device grade, thermally grown $\mathrm{SiO}_{2}$ films, containing fewer defects, exhibit an equal electronic sputter rate as the defect-rich, rf sputtered $\mathrm{SiO}_{2}$ films.

Below we make clear how the steep variation of the sputter rate at about $x=1.2$ has its origin in the valence band structure of the suboxides, in its associated charge transport, and in its evolution with varying $x$ [21-24].

In $\mathrm{SiO}_{x}$ the conduction band consists of both $\mathrm{Si}-\mathrm{Si}$ and $\mathrm{Si}-\mathrm{O}$ antibonding states, and these are positioned at more or less the same energy. Consequently, with increasing $x$ the position of the conduction band remains practically unaltered. For $x<1.5$ the valence band edge consists of SiSi bonding states. With increasing $x$ these states are more and more annihilated by the incorporated $\mathrm{O}$ atoms, forming $\mathrm{Si}-\mathrm{O}$ bonding states, positioned deep within the valence band. With increasing $x$ the Si-Si bonding states get more and more localized due to the appearance of more and more Si-O bonds. For $x$ increasing from 0 to about 1.5, this results in a gradual lowering of the valence band edge [25]. These Si-Si bonds represent delocalized states as long as interconnected chains of approximately $10 \mathrm{Si}$ atoms re-
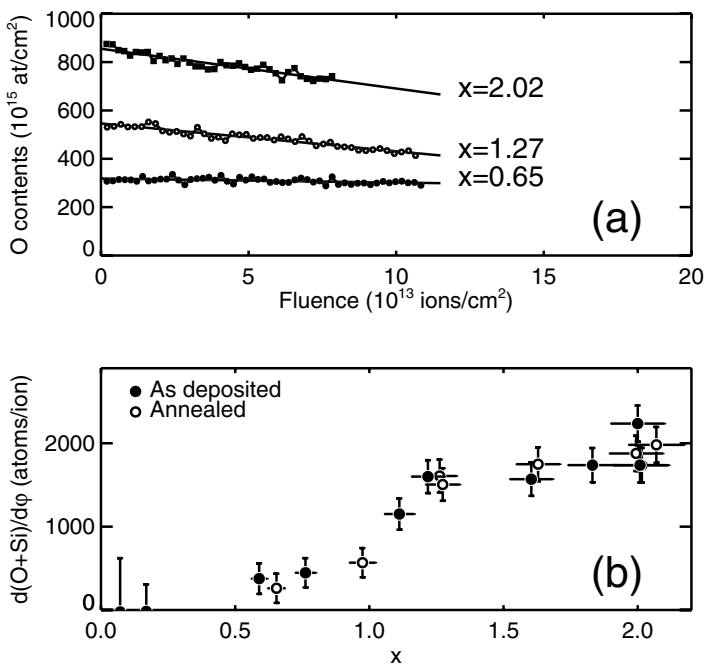

FIG. 2. (a) Total number of $\mathrm{O}$ atoms in the $\mathrm{SiO}_{x}$ layers as a function of the $50 \mathrm{MeV}$ ion fluence for $\mathrm{SiO}_{x}$ films for three different values of $x$. (b) $(\mathrm{O}+\mathrm{Si})$ removal rate during $50 \mathrm{MeV}$ ion irradiation from as-deposited and annealed $\mathrm{SiO}_{x}$ films as a function of $x$. 
main present in the compound [22,23]. Assuming a random bonding model for $\mathrm{SiO}_{x}$, this criterion is met in compounds with $x<1.5$. At higher $x$ values the Si-Si bonding states are too isolated to be considered delocalized, and the valence band edge drops to the energy level of the Si-O states.

The variation of the electronic structure with the variation of $x$ is reflected in the variation of charge transport properties in the material. The localization of the valence band states is accompanied by a strong decrease of the hole mobility for increasing $x$. The decrease is from the range $10^{-4}-10^{-3} \mathrm{~cm}^{2} / \mathrm{V}$ s or larger in $a-\mathrm{Si}[26,27]$ down to the range $10^{-5} \mathrm{~cm}^{2} / \mathrm{V} \mathrm{s}$ in $\mathrm{SiO}_{2}$ [28]; both values are quoted for room temperature. Obviously, since the hole mobility is much lower in $\mathrm{SiO}_{2}$ than in $a-\mathrm{Si}$, the decrease of the hole mobility of the valence band associated with the $\mathrm{Si}-\mathrm{Si}$ states, for increasing $x$, is not compensated for by an increase of the hole mobility in the Si-O valence band. The steepest variation is expected to occur around an $x$ value slightly below 1.5 where the $\mathrm{Si}$-Si states become localized at increasing $x$. Likewise, the strongly decreased hopping conduction for electrons in band gap states at about $x=1.2$ has been attributed to a stronger localization of these band gap states at this $x$ value [20]. In this Letter we show that the extent of localization of the states through which charge transport takes place determines whether or not significant sputtering takes place.

For the discussion of the $x$ dependence of the sputter yield, we first consider the electronic energy deposition along the ion trajectory in the solid. Evaluation of the stopping power in $\mathrm{SiO}_{x}$ as a function of $x$, using Bragg's rule and of the electronic excitation energy density as a function of $x$, estimated according to the method of Matsunami et al. [13], makes us deduce that the amount or density of the energy deposition along the track does not determine the sputter rate in the present conditions: whereas the stopping power only slightly varies with $x$, the deposited energy density [13] increases significantly only for $1.5<x<2$, where the track diameter decreases because of the strong increase of the band gap [25]. The increase in the observed sputter yield in this regime of $x$ values, however, is relatively small.

Recently, Bringa and Johnson performed molecular dynamics (MD) simulations of Coulomb explosion sputtering for cylindrical tracks as a function of charge density and neutralization time in Lennard-Jones fcc solids [4]. It appeared that the sputter yield increases with increasing neutralization time to appreciable values when the neutralization time is in the order of the Debye period, which amounts to $\sim 10^{-13} \mathrm{~s}$, but tends to become constant for larger track lifetimes. In accordance with these MD simulations we hypothesize that the track lifetime increases with increasing $x$, and in the region $1.1<$ $x<1.5$ it reaches the value of the Debye period. Consistently, a further increase of the lifetime for increas- ing $x$ has no large consequences for the sputter yield [see Fig. 2(b)].

For a general discussion of time scales we refer to the well-known table of time scales in high energy ion tracks of Ritchie and Clausen for $1 \mathrm{MeV}$ He in solid Ar [29]. At first, ejection of the (so-called) $\delta$ electrons occurs in a time of $\sim 10^{-17}$ s. Energetic $\delta$ electrons (i.e., $E_{\text {kin }} \gtrsim 10 \mathrm{eV}$ ) have an inelastic mean free path (IMFP) of at most a few $\mathrm{nm}$ [30], because they are inelastically scattered by impact ionization and plasmon excitation, thereby decreasing their kinetic energy down to an energy below the band gap energy. This process takes place within $\sim 10^{-14} \mathrm{~s}$. It is expected that most of the electrons do not leave the track region because of the Coulomb attraction and because of the small IMFP. So, at about $10^{-14} \mathrm{~s}$ after the ion passage, we have a track of positive ions and many low-energy electrons, which move in the track potential.

One can speak of a track potential as long as the electrons are distributed over a larger volume than the positive ions, which results in a net positive charge along the axis of the cylinder and a net negative charge at the track surroundings [31]. In the next step, electrons lose further kinetic energy. For $\mathrm{SiO}_{2}$ this is likely to take place predominantly by electron-phonon scattering. This process has been calculated to be by far the fastest $\left(\sim 10^{-14} \mathrm{~s}\right)$ at very low kinetic energies [32], and therefore is expected to take place preferentially at the track edge. This results in trapping of electrons at the track edge and thus in a net negative charge buildup in a cylindrical shell around a positively charged core. It should be noted that for the system under study relaxation by radiative processes occurs on a much longer time scale of ns [33] and is not expected to play any role of importance. In this manner, to reach this state of separation of net positive charge at the center and negative charge at the edge of the track cylinder will take several steps of $10^{-14} \mathrm{~s}$ amounting to a time less than about $10^{-13} \mathrm{~s}$. The essential step in our model is that subsequent track neutralization takes place through transport of electrons via (localized) band gap states and through drift of holes from inside the track. In this process the $x$ dependence becomes manifest. As discussed before, for $x<1.2$ hole transport is fast. For larger $x$, track neutralization takes place through hopping of trapped holes and electrons because of the increased extent of localization of relevant states. The hopping attempt frequency is in the order of the phonon frequency of $10^{13} \mathrm{~s}^{-1}$. Since several consecutive hopping steps are needed to neutralize the track, its lifetime is principally above the Debye period of $10^{-13} \mathrm{~s}$. In this way it is conceivable that the steep increase in localization of the states involved in hole and electron hopping transport in the transition region causes the track lifetime to increase from below the Debye period to significantly above the Debye period. The latter long track lifetime results in sputtering due to a net Coulomb repulsion in the ion track, as has been computed by MD 
simulations [4]. Consequently, and this is the main result of this Letter, we identify the drift of holes from the track and/ or transport of trapped electrons to the track as sputter rate determining processes in this class of materials.

Our model is consistent with the experiments by Ninomiya and Imanishi [34], who observed the emission of positively charged species having an energy of about $10 \mathrm{eV}$ or larger, linearly increasing with the charge state of the detected species. This fits in the picture of positive ions getting accelerated by the Coulomb track. The model that electronic sputtering would be simply evaporation of species in the thermal spike [3] is not supported by these experimental observations. The presented model is also consistent with recent experiments in which it was observed that a $\sim 1 \mathrm{~nm}$ layer of $\mathrm{SiO}_{2}$, when present on $\mathrm{Si}$, cannot be removed by electronic sputtering [17]. If the surface of the track is within the tunneling distance of a (semi)conducting layer, charge transport is fast, the track lifetime is reduced, and electronic sputtering virtually stops. Indeed, when the $\mathrm{SiO}_{2}$ layer was deposited on an insulator $\mathrm{Si}_{3} \mathrm{~N}_{4}$ covered $\mathrm{Si}$ substrate, it could be completely removed by sputtering with swift heavy ions [17].

The process of electronic sputtering by swift heavy ions may be similar to the process of potential sputtering as a result of slow highly charged ions [12]. Potential sputtering of $\mathrm{SiO}_{2}$ has been suggested to occur invoking self-trapped excitons (STE) [11]. The observed $x$ dependence of the removal rate in $\mathrm{SiO}_{x}$ does not seem to support such an STE mechanism in $\sim \mathrm{MeV} /$ a.m.u. ion sputtering since one would then expect a larger dependence of the sputter rate on $x$ for $x>1.5$. The existence of STE's, however, and the model presented here may well be essentially related since in both cases holes and excited electrons localized on a nanometer scale are involved.

To summarize, we have shown how the experimentally observed $x$ dependence of the electronic sputter yield in $\mathrm{SiO}_{x}$ can be understood from the lifetime of the charged track considering the track neutralization mechanisms, the electronic structure of the material, and the associated charge transport. The results of the experiments are consistent with the general features of recently published MD simulations of Coulomb explosion sputtering.

[1] M. Sporn, G. Libiseller, T. Neidhart, M. Schmid, F. Aumayr, HP. Winter, P. Varga, M. Grether, D. Niemann, and N. Stolterfoht, Phys. Rev. Lett. 79, 945 (1997).

[2] W. Marine, N. M. Bulgakova, L. Patrone, and I. Ozerov, Appl. Phys. A 79, 771 (2004).

[3] M. Toulemonde, W. Assman, C. Trautmann, F. Grüner, H. D. Mieskes, H. Kucal, and S. G. Wang, Nucl. Instrum. Methods Phys. Res., Sect. B 212, 346 (2003).

[4] E. M. Bringa and R.E. Johnson, Phys. Rev. Lett. 88, 165501 (2002).
[5] R.E. Johnson and B. U.R. Sundqvist, Phys. Today 45, No. 3, 28 (1992).

[6] R. E. Johnson and J. Schou, Mat. Fys. Medd. K. Dan. Vidensk. Selsk. 43, 403 (1993).

[7] M. Beuve, N. Stolterfoht, M. Toulemonde, C. Trautmann, and H. M. Urbassek, Phys. Rev. B 68, 125423 (2003).

[8] C.H.M. Marée, A. M. Vredenberg, and F.H.P.M. Habraken, Mater. Chem. Phys. 46, 198 (1996).

[9] R. L. Fleischer, P. B. Price, and R. M. Walker, J. Appl. Phys. 36, 3645 (1965).

[10] R.E. Johnson, B. U. R. Sundqvist, A. Hedin, and D. Fenyö, Phys. Rev. B 40, 49 (1989).

[11] F. Aumayr, J. Burgdörfer, G. Hayderer, P. Varga, and HP. Winter, Phys. Scr. T80, 240 (1999).

[12] Friedrich Aumayr and Hannspeter Winter, Phil. Trans. R. Soc. A 362, 77 (2004).

[13] N. Matsunami, M. Sataka, and A. Iwase, Nucl. Instrum. Methods Phys. Res., Sect. B 193, 830 (2002).

[14] M. Matsunami, M. Sataka, A. Iwase, and S. Okayasu, Nucl. Instrum. Methods Phys. Res., Sect. B 209, 288 (2003).

[15] E. M. Bringa, Nucl. Instrum. Methods Phys. Res., Sect. B 209, 1 (2003).

[16] S. Sugden, C. J. Sofield, and M. P. Murell, Nucl. Instrum. Methods Phys. Res., Sect. B 67, 569 (1992).

[17] W. M. Arnoldbik, N. Tomozeiu, and F. H. P. M. Habraken, Nucl. Instrum. Methods Phys. Res., Sect. B 203, 151 (2003).

[18] W. M. Arnoldbik, N. Tomozeiu, and F. H. P. M. Habraken, Nucl. Instrum. Methods Phys. Res., Sect. B 190, 433 (2002).

[19] J. J. van Hapert, A. M. Vredenberg, E. E. van Faassen, N. Tomozeiu, W. M. Arnoldbik, and F. H.P.M. Habraken, Phys. Rev. B 69, 245202 (2004).

[20] J. J. van Hapert, Ph.D. thesis, Utrecht University (2002).

[21] F. G. Bell and L. Ley, Phys. Rev. B 37, 8383 (1988).

[22] P. Ordejón and F. Ynduráin, Phys. Rev. B 43, R4552 (1991).

[23] E. Martinez and F. Ynduráin, Phys. Rev. B 24, 5718 (1981).

[24] W. Y. Ching, Phys. Rev. B 26, 6633 (1982).

[25] E. Holzenkämpfer, F.-W. Richter, J. Stuke, and U. VogetGrote, J. Non-Cryst. Solids 32, 327 (1979).

[26] A. R. Moore, Appl. Phys. Lett. 31, 762 (1977).

[27] P. G. Lecomber, A. Madan, and W. E. Spear, J. Non-Cryst. Solids 11, 219 (1972).

[28] R. C. Hughes, Phys. Rev. B 15, 2012 (1977).

[29] R.H. Ritchie and C. Clausen, Nucl. Instrum. Methods Phys. Res. 198, 133 (1982).

[30] J.-Ch. Kuhr and H.-J. Fitting, J. Electron Spectrosc. Relat. Phenom. 105, 257 (1999).

[31] B. Gervais and S. Bouffard, Nucl. Instrum. Methods Phys. Res., Sect. B 88, 355 (1994).

[32] R. Ludeke, A. Bauer, and E. Carter, J. Vac. Sci. Technol. B 13, 1830 (1995).

[33] NIST Atomic Spectra Database Lines Data, http:// physics.nist.gov/cgi-bin/AtData/lines_form.

[34] S. Ninomiya and N. Imanishi, Vacuum 73, 79 (2004). 